\title{
Pityriasis rosea like eruption secondary to topiramate: case report
}

\begin{abstract}
Drug related cutaneous eruptions are everyday medical practice, so they should always be remembered as a differential diagnosis. Peculiar presentations, mimicking conditions other than drug-induced, add difficulty to the diagnosis. In this context, it can be mentioned pityriasis rosea like eruption secondary to drugs, and its consideration as a hypothesis in the differential diagnosis gains importance in the sense that while in contact with the drug, unlike idiopathic pityriasis rosea, the rash does not show spontaneous resolution. We present a case of pityriasis rosea like triggered by topiramate, seeking to differentiate this condition from idiopathic pityriasis rosea, according the literature.
\end{abstract}

Mesh terms: drug eruption, pityriasis rosea, topiramate
Volume 2 Issue 4 - 2018

\author{
Gustavo Moreira Amorim, ${ }^{1,2}$ Gabriel \\ Amorim, ${ }^{2}$ Dâmia Leal Vendraminin Amorim,' \\ Danielle Carvalho Quintella, 'Tullia Cuzzi, ${ }^{1,3}$ \\ 'Federal University of Rio de Janeiro, Brazil \\ 2Universidade Do Sul De Santa Catarina, Brazil \\ ${ }^{3}$ Instituto de Dermatologia Professor Ruben David Azulay, Brazil
}

Correspondence: Gustavo Moreira Amorim, Federal

University of Rio de Janeiro, Brazil,

Email gustavomoreiraamorim@hotmail.com

Received: April 17, 2018| Published: July 12, 2018

\section{Introduction}

Pityriasis rosea (PR) is a common papulosquamous eruption that affects mainly young healthy adults with a slight female predominance and a characteristic seasonality with peaks in spring and fall. ${ }^{1}$ Described by Camille Melchior Gilbert, in 1860, in its classical form has an exuberant cutaneous presentation, but still presents a benign and selflimited clinical course, resolving spontaneously within 6-8weeks. Its exact cause remains unknown, and among the studied hypotheses, the most accepted is a possible viral etiology determined by human herpes virus 7 and $6.1,2$

It is known that skin involvement in adverse drug reactions is common, so the possibility of adverse effect of a medicine should be always considered in a cutaneous eruption. Especially if we take into account the ability of these adverse reactions mimic common clinical conditions not classically associated with drugs. A PR like eruption, triggered by drugs, mimicking classic pityriasis rosea is an example of this situation. ${ }^{3}$

Have already been implicated gold salts, inhibitors of angiotensinconverting enzyme, metronidazole, isotretinoin, beta blockers, barbiturates, sulfasalazine, bismuth, clonidine, imatinib mesylate, arsenic, interferon, among others. Besides the drugs, vaccination with BCG and hepatitis B also were described as causes. ${ }^{4}$

The recognition of this entity is important in the differential diagnosis with classical PR, since identifying a possible drug as cause allows it suspension, enablesarapidclinicalimprovement.

\section{Reason for communication}

We report a case of drug eruption PR like, trying to demonstrate their differences in relation to the classical form and the importance of correct diagnosis for appropriate management of the patient and his complaint skin.

\section{Case report}

Male patient, 53years old, caucasian, retired, born and raised in Rio de Janeiro, comes in consultation due to slightly erythematous patches on the thighs, emerged 45 days earlier, with progressive growth and appearance of new lesions. He denied pruritus or other symptoms and reported that he had initiated topiramate as part of the treatment of obesity 2 months ago. On examination, oval erythematous plaques with mild scaling and slightly raised edges present were seen in posterior face of both thighs, one being bigger than the others, with about $4.0 \mathrm{cmin}$ diameter (Figure 1) (Figure 2). Microscopic examination after $\mathrm{KOH}$ preparation and culture of the scrapings from cutaneous lesions were negative. Blood count and biochemistry also normal. The assumption made was a pityriasis rosea like induced by drug. Biopsy of the lesion showed perivascular and slight lichenoid dermatitis with extravasation of red blood cells, confirming the hypothesis by histopathological correlation. With about 12 weeks of evolution, we orient the suspension of the topiramate and the patient progressed presented good response, retaining only residual hyperpigmentation after 15 days .

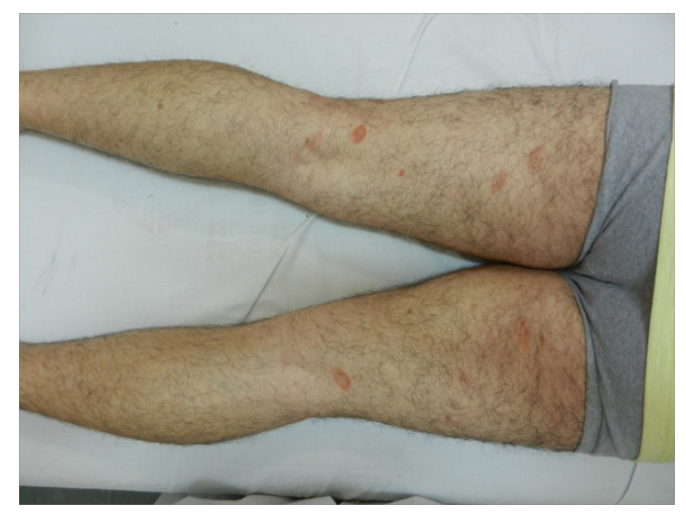

Figure I Oval erythematous plaques on lower limbs 


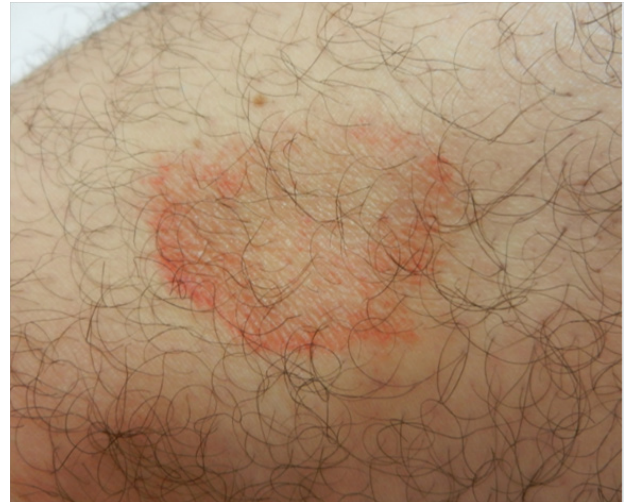

Figure 2 Fine scales with slightly elevated edges

\section{Discussion}

In terms of epidemiology it is found a male predominance, with 50 years old or more in the PR like rash secondary to drugs, unlike the idiopathic PR. ${ }^{3}$

The clinical features may be quite similar to the idiopathic PR, with papules or oval erythematous plaques with raised borders and central yellowish scaly. Between 50 and $90 \%$ of the patients with idiopathic RP present a single lesion, well-defined, oval, preceding a diffuse rash with smaller lesion within 14days. ${ }^{4}$ Apparently, this doesn't occur in most reported cases of PR simile, where the rash starts or progresses faster to dissemination. ${ }^{3}$ Also in relation to the lesions characteristics, the eruption triggered by drug presents more intense erythematous lesions, some even tending to violet, and a greater exuberance in terms of length and number of lesions. Furthermore, the distribution pattern of the lesions in idiopathic PR, especially affecting the trunk and root of the members, is not always detected in the PR like eruption. Apparently, extremities are affected in a asymmetric way.

Fever seems to be more frequente in drug related cases and itching can be intense, generally refractory to antihistamines. ${ }^{3}$

The time of lesions appearance in relation to treatment initiation with the drug in question is on average from 5 to 20days. Another fact that points to the possibility of drug induced PR is more extended course. While most of the references describe a self-limiting course, with mean duration of 6 to 8 weeks for the PR idiopathic, the drug eruption can be prolonged and lasts as long as the patient maintains the use of the medication. Stopping the drug, is expected a resolution around 5 to 10days. In remission, post-inflammatory hyperpigmentation is observed more often in PR like. ${ }^{3}$

An increased level of eosinophils in peripheral blood may be found when we are faced with a case of PR like, however this finding beyond insensitive, is rather nonspecific. ${ }^{3}$

Although the diagnosis in some studies consulted, has been defined with retrospective clinical improvement of symptoms with medication withdrawal, histopathological examination may be valuable. Characteristics findings of classical PR Idiopathic can be seen, such as focal parakeratosis, acanthosis, exocytosis and spongiosis, extravasation of erythrocytes, both permeating the epithelium and in the dermis. Superficial perivascular dermatitis, lympho- monocytic and eosinophilic infiltrate point to drug induced rash.,

Cutaneous eruptions drug related are everyday medical practice, so that should always be remembered as a differential diagnosis. Peculiar presentations, mimicking conditions other than drug-induced, add difficulty to the diagnosis. The only data in terms of frequency that we found in our literature review was a prospective study conducted by a group of European surveillance ofdrugs, that found $\%$ ofalldrugrashes manifested as PR like. ${ }^{3}$ Still, the authors conclude that these conditions are probably underdiagnosed, contributing for the low frequency.

The anticonvulsant drug topiramate is indicated for treatment of epilepsy, either as monotherapy or as adjuvant. It is also effective in the prophylaxis of migraine. Furthermore, neurogenic pain control and treatment eating disorders as an adjuvant are off label indications. ${ }^{6}$ Its cutaneous side effects are considered unusual and when they occur, nonspecific rash, localized edema and diffuse pruritus are described as more frequent.

We didn't found no other studies reporting the association of topiramate and PR like eruption, however the greatest importance of the report lies in the fact that the misdiagnosis of idiopathic PR could delay the suspension of the drug, probably prolonging the clinical course and increasing patient morbidity. Finally, draw attention to this entity.

\section{Acknowledgements}

None.

\section{Conflict of interest}

The author declares no conflict of interest.

\section{References}

1. Ayanlowo O, Akinkugbe A, Olumide Y. The pityriasis rosea calendar: a 7 year reviewofseasonalvariation, ageandsex distribution. Nig QJHosp Med. 2010;20(1):29-31.

2. Chuh A, Lee A, Zawar V, et al. Pityriasis rosea-an update. Indian J Dermatol Venereol Leprol. 2005;71(5):311-315.

3. Atzori L, PinnaAL, FerreliC, et al. Pityriasis rosea-like adverse reaction: Review of the literatureandexperienceofanItaliandrug-surveillancecenter. Dermatol Online J. 2006;12(1):1.

4. Stulberg DL, Wolfrey J. Pityriasis rosea. Am Fam Physician. 2004;69(1):8791.

5. Lai YW, Chou CY, Shen WW. Pityriasis rosea-like eruption associated with clozapine: a case report. Gen Hosp Psychiatry. 2012;34(6):703.e5-7.

6. Lin K. Topiramato: uma molécula multifacetada. Rev Neurocienc. 2011;19(1):8-9. 